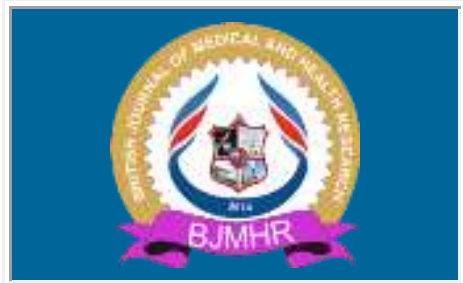

\title{
BJMHR
}

British Journal of Medical and Health Research Journal home page: www.bjmhr.com

\section{Correlation of Diagnostic Accuracy of ELISA and Rapid Test Reader Used In Immunochromatographic Assay}

Kathiravan $\mathbf{V} *$, Swaminathan $\mathbf{S}$

Department of Microbiology, TechMed Healthcare, Chennai, India.

\begin{abstract}
Early and accurate detection of HIV infection is essential for patient management and disease control. The primary methodology for HIV testing has shifted from enzyme linked immunosorbent assay (ELISA) to rapid diagnostic tests in recent years, especially in resource limited settings. The study correlate two new commercial serologic tests for HIV (ALTA rapid test reader RTR-1, CTK Biotech and HIV ELISA; Genscreen ${ }^{\mathrm{TM}}$ ULTRA HIV Ag-Ab). The sensitivity and specificity of the HIV tests were compared using unknown HIV status of 955 specimens. The two different assays showed excellent sensitivity in the diagnosis of HIV infection (100\%). Specificity in RTR was (100\%), whereas it is $(98.3 \%)$ in ELISA which indicates, ELISA given (1.7\%) false positive results. Rapid test reader can be useful in the effective and economical screening method for HIV infection. However, rapid test reader is not a substitute for ELISA method
\end{abstract}

Keywords: HIV diagnosis, ELISA, Rapid diagnostic test reader 


\section{INTRODUCTION}

According to UNAIDS report 2018, in India 2.1 million people are living with HIV and only 2 million people were known their status.(1) Compared to neighbouring countries, India has made good progress in reducing new HIV infections by a half since 2001.(2) Early knowledge of HIV status of an individual is the key for HIV prevention and therapeutic intervention.

Enzyme linked immunosorbent assay (ELISA) is preferred and recommended screening technique in detection of HIV antibodies as marker of HIV exposure.(3) The intense of labour involved and the time-consuming factor of ELISA assay and also the requirement of technical expertise has urged researchers to do research to bring shift from an ELISA assay to other simple diagnostic tests particularly in resources constrained settings.(4)

Emerging business setups on medical field has increased the burden on common man's shoulder especially in developing countries. In India, doctors are now insisting on HIV tests for all surgery cases because patients are not willing to disclose their health problem before undergoing surgery. Tests are compulsorily done at all private hospitals so that doctors can be safe.(5)

Ideally, testing for $\mathrm{HIV}, \mathrm{HBsAg}$ and $\mathrm{HCV}$ should be mandatory for elective surgeries in particular, especially in areas where the diseases are prevalent. But it may not be cost- effective to do them routinely before surgery in most developing countries. In this scenario, researchers are pushed to do studies on cost effective and accurate ways for screening of infectious diseases.

Some studies have reported the performance of rapid diagnostic tests and ELISA to be comparable.(6) Results from all these researches have raised the concern about the sensitivity and specificity of rapid diagnostic assays. But the correlation between HIV rapid test reader (RTR) results and the serologic detection of HIV p24 antigen and antibodies to HIV-1 and HIV-2 with a new-generation ELISA has not been investigated. (7-9)

The rapid test reader (RTR) is designed to analyse and diagnose target disease by using existing consumer cameras of a laptop-computer or a tablet. The RTR is useable with numerous lateral immunochromatographic assays. The device has a programme that takes the image of rapid strips, crops the region of interest, and extracts the features from the control end test lines to classify the results as invalid, positive, or negative.

The aim of the study was to analyse the diagnostic efficacy of RTR and its correlation with ELISA results in unknown HIV status of 955 serum samples in TechMed Laboratory, Chennai.

\section{MATERIALS AND METHOD}

The study was performed from August 21, 2018, to September 10, 2018, doing comparison of results obtained from ELISA and RTR. The rapid detection strips were read and the results 
were interpreted by ALTA rapid test reader device. The study was conducted at the TechMed Laboratory, a leading hospital laboratory management service provider in Chennai, Tamil Nadu, India.

The present study included 955 patient serum samples which were received from different hospital laboratory management sectors of TechMed. All the samples received were actually collected from patients for routine laboratory examination and not purposely for HIV testing. And hence, written informed consent from patient for HIV testing was not obtained.

Screening for HIV was performed with a next generation ELISA (Genscreen ${ }^{\mathrm{TM}}$ ULTRA HIV Ag-Ab) and a RTR (ALTA rapid test reader RTR-1, CTK Biotech). The ELISA kit detects HIV p24 antigen and antibodies to HIV-1 and HIV-2 in human serum or plasma by enzyme immunoassay. The ALTA rapid test reader is an image-analysis based qualitative/semiquantitative reader specifically designed to measure and interpret lateral flow immunoassay (immunochromatography) test results. The rapid test reader device has camera with its twodimensional CCD image sensor, which does not only scan the rapid test strip line-by- line, but also records a complete image of the test strip, which is not only an advantage in quantitative analysis, but also makes an optimal interpretation of the test results possible.

Cut off values were used to define a positive ELISA result and RTR result. A cut off value of 0.290 or more is consider a positive for ELISA and a cut off value of 15 or more is considered a positive for RTR.

The statistical analysis was performed using IBM SPSS Statistics. The Pearson correlation coefficient ( $\mathrm{r}$ ) was calculated to evaluate the correlation between the results obtained by ELISA and rapid test reader. Furthermore, the sensitivity, specificity, false positive, and false negative for the detection of HIV infection by ELISA and rapid test reader were obtained, using the manually interpreted rapid test results (qualitative) as reference

\section{RESULTS AND DISCUSSION}

With ELISA reactivity defined as a cut off value of 0.290 or more, 929 (97.3\%) samples tested negative by ELISA; all these samples also detected negative by RTR. 26 (2.7\%) samples had a positive ELISA result. Of these 26 samples only 10 had a positive RTR result $\left(r=0.804^{* *}\right)$.

Table 1: Results on follow-up of all patients with a positive ELISA results (cut off $>0.290$ ) and a positive RTR results (cut off $>15$ )

\begin{tabular}{llll}
\hline Sample & ELISA value & RTR value & HIV rapid result \\
\hline 1 & 4.00 & 25.9 & Positive \\
2 & 3.68 & 24.0 & Positive \\
3 & 3.08 & 21.0 & Positive \\
4 & 2.88 & 26.8 & Positive \\
5 & 2.68 & 31.3 & Positive \\
6 & 0.43 & 15.3 & Positive \\
\hline
\end{tabular}




\begin{tabular}{llll}
\hline 7 & 2.24 & 23.9 & Positive \\
8 & 1.00 & 22.0 & Positive \\
9 & 0.48 & 16.0 & Positive \\
10 & 3.53 & 33.3 & Positive \\
11 & 0.30 & 3.40 & Negative \\
12 & 0.41 & 5.30 & Negative \\
13 & 0.41 & 4.00 & Negative \\
14 & 0.30 & 0.00 & Negative \\
15 & 0.32 & 6.90 & Negative \\
16 & 0.55 & 0.00 & Negative \\
17 & 0.37 & 2.30 & Negative \\
18 & 0.30 & 2.50 & Negative \\
19 & 0.32 & 0.30 & Negative \\
20 & 0.34 & 2.30 & Negative \\
21 & 0.36 & 0.00 & Negative \\
22 & 0.48 & 7.00 & Negative \\
23 & 0.34 & 0.20 & Negative \\
24 & 0.29 & 0.00 & Negative \\
25 & 0.31 & 0.30 & Negative \\
26 & 0.54 & 00.0 & Negative \\
\hline
\end{tabular}

Totally, 10 (1\%) samples had an HIV infection confirmed by HIV rapid (qualitative) method. None of these samples tested negative by HIV RTR device. The sensitivity, specificity, false positive and false negative values for the detection of HIV infection by ELISA and RTR are provided in (Table.2).

Table 2 Diagnostic accuracy of the ELISA and HIV RTR, with HIV rapid as reference method

\begin{tabular}{lllll}
\hline Test & Sensitivity, \% & Specificity, \% & False Positive, \% & False Negative, \% \\
\hline ELISA & 100 & 98.3 & 1.7 & 0 \\
HIV RTR & 100 & 100 & 0 & 0 \\
\hline
\end{tabular}

\section{DISCUSSION}

Various studies proved HIV rapid testing is an effective test for the detection of HIV infection. In a study conducted in Netherlands, HIV rapid test was used to evaluate 1160 patients' sample to test for HIV type 1 for quick clinical decision making, such as in needle-stick accidents. The test proved to be a simple and useful test with $99.6 \%$ specificity and $99.4 \%$ sensitivity.(10)

The rapid test used in the study as a reference method correlated efficiently with new generation ELISA and HIV rapid test reader and the test identified well the samples with a false positive ELISA result.

All around the world, researches were done to find a substitute for HIV ELISA screening which is cost effective also. A study done in Santiago, Chile: to a total of 497 people, 153 with known HIV status and 344 people with unknown HIV status, were tested for HIV using both ELISA and oral rapid test to examine and compare specificity and sensitivity. In the end result, both 
were statistically equal in specificity and sensitivity and also as an advantage oral rapid test provides quicker results, and does not require handling of or exposure to potentially hazardous blood products.(4)

From the study conducted, we come to know that the rapid test reader also interprets results on quantitative basis. The results are given based on the colour intensity of the band that is present inside the strip. Also, the test provides some advantages like time consumption and cost effective and accurate results when compared with an ELISA screening.

Studies shows that there are many critical factors affecting the diagnostic reliability of ELISA formats.(11) In a study conducted in Spain, the influence of sample cut off (S/CO) values by a 4th-gen-ELISA with the false positive rate during the routine HIV diagnosis in a low HIV prevalence population was evaluated. Among the 30,201 HIV performed tests, $256(0.85 \%)$ were positive according to ELISA interpretation $(\mathrm{S} / \mathrm{CO} \geq 1)$ but only $229(0.76 \%)$ were definitively HIV-1 positive after confirmatory or reference tests. The study concludes that the false positive rate is reduced when cut off increases.(12)

In the present study also, the low cut off value defined for ELISA result interpretation affects the diagnostic accuracy. The sensitivity of the ELISA in screening the reactive samples is hundred percent but the specificity is affected.

The present study also has some limitations. The reference method that is used is the rapid tridot method. Although, test performers were properly trained, but the chances of human error in interpreting results remains.

\section{CONCLUSION}

The HIV RTR results showed adequate correlation with a standard new generation ELISA. Also, the study shows that very low cut off during HIV screening using Genscreen can result HIV negative after confirmation by RTR. And rapid test reader can be useful in the effective and economical screening method for HIV infection. However, rapid test reader is not a substitute for ELISA method.

\section{ACKNOWLEDGMENTS}

The author would like to express his sincere gratitude to Prof. Dr S Swaminathan, Director, TechMed Laboratory for his encouragement and guidance in completing this work. Authors would like to thank Techmed Lab management and its associate vice president - Dr Masood Ikram for the necessary support rendered and also thankful to the officials and other staff members of Techmed who rendered help during the period of the work. 


\section{REFERENCES}

1. Global HIV \& AIDS statistics — 2018 fact sheet [Internet]. [cited 2019 Feb 22]. Available from: http://www.unaids.org/en/resources/fact-sheet

2. HIV and AIDS in India [Internet]. AVERT. 2015 [cited 2019 Feb 22]. Available from: https://www.avert.org/professionals/hiv-around-world/asia-pacific/india

3. Torane VP, Shastri JS. Comparison of ELISA and rapid screening tests for the diagnosis of HIV, Hepatitis B and Hepatitis C among healthy blood donors in a tertiary care hospital in Mumbai. Indian J Med Microbiol. 2008 Sep;26(3):284-5.

4. Irarrazábal LP, Ferrer L, Cianelli R, Lara L, Reed R, Levy J, et al. Oral rapid test: an alternative to traditional HIV screening in Chile. Rev Panam Salud Publica Pan Am J Public Health. 2013 Jun;33(6):427-32.

5. Gokul R. Hospitals insist on HIV test before surgery - Times of India [Internet]. The Times of India. [cited 2019 Feb 22]. Available from: https://timesofindia.indiatimes.com/city/trichy/Hospitals-insist-on-HIV-test-beforesurgery/articleshow/34444283.cms

6. Lien TX, Tien NT, Chanpong GF, Cuc CT, Yen VT, Soderquist R, et al. Evaluation of rapid diagnostic tests for the detection of human immunodeficiency virus types 1 and 2, hepatitis B surface antigen, and syphilis in Ho Chi Minh City, Vietnam. Am J Trop Med Hyg. 2000 Feb;62(2):301-9.

7. Claassen M, van Zyl GU, Korsman SNJ, Smit L, Cotton MF, Preiser W. Pitfalls with rapid HIV antibody testing in HIV-infected children in the Western Cape, South Africa. J Clin Virol Off Publ Pan Am Soc Clin Virol. 2006 Sep;37(1):68-71.

8. Gray RH, Makumbi F, Serwadda D, Lutalo T, Nalugoda F, Opendi P, et al. Limitations of rapid HIV-1 tests during screening for trials in Uganda: diagnostic test accuracy study. BMJ. 2007 Jul 28;335(7612):188.

9. Louie B, Wong E, Klausner JD, Liska S, Hecht F, Dowling T, et al. Assessment of Rapid Tests for Detection of Human Immunodeficiency Virus-Specific Antibodies in Recently Infected Individuals. J Clin Microbiol. 2008 Apr 1;46(4):1494-7.

10. van den Berk GEL, Frissen PHJ, Regez RM, Rietra PJGM. Evaluation of the Rapid Immunoassay Determine HIV $1 / 2$ for Detection of Antibodies to Human Immunodeficiency Virus Types 1 and 2. J Clin Microbiol. 2003 Aug;41(8):3868-9. 
11. Schrijver RS, Kramps JA. Critical factors affecting the diagnostic reliability of enzymelinked immunosorbent assay formats. Rev Sci Tech Int Off Epizoot. 1998 Aug;17(2):550-61.

12. Chacón L, Mateos ML, Holguín Á. Relevance of cutoff on a 4th generation ELISA performance in the false positive rate during HIV diagnostic in a low HIV prevalence setting. J Clin Virol Off Publ Pan Am Soc Clin Virol. 2017;92:11-3.

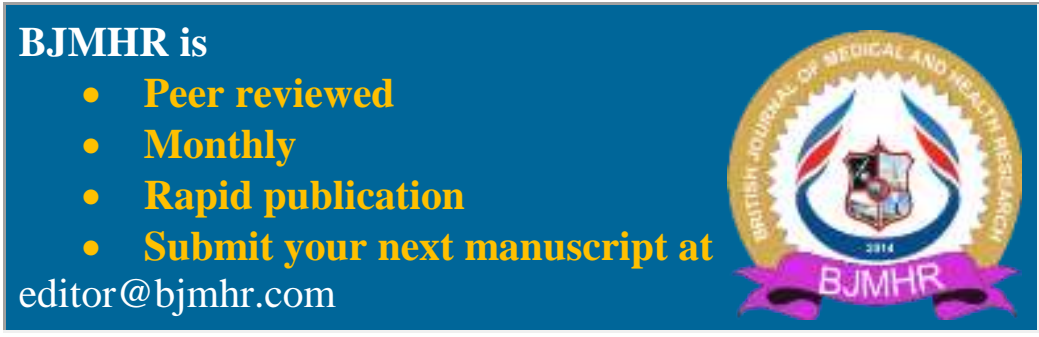

